\title{
Genetic analysis of DXS101and DXS6807 short tandem repeat (X-STR) in samples of Iraqi Arab male population
}

\author{
Hayder Allawi Khaleefah Salwa Jaber Abdullah Al-Awadi Zaid Nsaif Abbas Al-Tameemi \\ College of biotechnology /AL-Nahrian University \\ E-mail: hayderallawi90@gmail.com
}

\begin{abstract}
:
Back ground: X-chromosomal short tandem repeats (X-STRs) have assured to be informative and particular role in complex relationship testing. DXS6807 known as tetra nucleotides polymorphism representing eight alleles of 251-275 bp in length. DXS6807 is located in, at XP 22.2, at a genetic distance of more than 87 and $151 \mathrm{Cm}$ of X-chromosome. DXS101 is located104.9-121 $\mathrm{cM}$ from the Xp-telomere
\end{abstract} (Xp-tel) corresponding to cytogenetic position in $\mathrm{Xq21.33-Xq22.3.}$

Objective: The aim of this present study investigates the allele frequency of two markers DXS101, DXS6807 and forensic efficiency parameters for sample of Arabic Iraqi males.

Material and methods: The population of this study includes 200 males apparently healthy unrelated participants from different region of Baghdad city, their ages ranged between (20-50) years. The Genomic DNA extracted and purified successfully from blood samples.

Results: The forensic efficiency parameters result for these markers were following: polymorphism information content (PIC) of $\mathbf{0 . 8 3 4 7 0 8}$, power of discrimination (PD) in male 0.851750 , Power of exclusion (PE) 0.698316, MEC Krüger0.511679, MEC Kishida 0.694890. The forensic efficiency parameters analyzing from Arabic population were Power of discrimination $(P D)=0.73405$, Polymorphism information content $(\mathrm{PIC})=\mathbf{0 . 6 9 4 8 9}$, Power of exclusion $(\mathrm{PE})=0.482879$.MEC Krüger $=0.511679$, MEC Kishida $=0.694890$.

Conclusions: The information provided establish this $\mathrm{X}$-linked microsatellite marker as a valuable strategy for forensic application. DXS101 is and DXS6807 recently consider more stable and suitable forensic markers for forensic application.

Keywords: Short tandem repeat, X-chromosome, Kinship, testing.

\section{Introduction:}

The short tandem repeat (STR) markers are typically scattered across the human genome and have been widely researched and applied to forensic analysis and identification of individuals. Most forensic issue was reporting of autosomal and Y-chromosomal STRs, but it is not widely possible to achieve the population genetic data for X-STR markers.because its usefulness in complex kinship research, X-STRs analysis had earlier attracted the attention of the forensic community. For cases where father / daughter relationships are to be checked, it is advantageous having X-STRs with autosomal markers (1). Because of their specific mode of genetic transmission of hemizygosity in male, X-chromosome STR markers (X-STR) may resolved forensic issues that do not reach the high levels of trust usually needed in legal proceedings, especially with regard to paternities with deficiencies (2). Furthermore, the study of X-STRs will significantly support the investigation 
of such complicated cases of kinship, like (paternal half-sisters. paternal aunt / uncle-niece), along with maternal uncle nephew (3). The DXS101 microsatellite marker which is a strongly polymorphic trinucleotide polymorphism with a length of 10 alleles from (198-222) bp. The Xp-telomere (Xp-Tel) DXS101 is situated (104.9-121) cM corresponding to the (Xq21. In 33-Xq22. 3). The information shown establish this X-linked microsatellite marker as a powerful option for forensic application (4). While HumDXS6807 is tandem repeat, it also recognizes the length of tetranucleotide repeat polymorphism alleles $(251-275$ bp $)$ as (CHLC.GATA52B03). HumDXS6807 is not linked to Hum ARA and Hum HPRTB, which are located at a genetic distance from this region ranged from $80 \mathrm{cM}$ and $150 \mathrm{cM}$, respectively. these three markers are appropriate for concomitant use in kinship testing and forensic application (5).

Materials \& Methods:

\section{Sample collection and DNA Extraction}

The population of this study includes 200 males apparently healthy unrelated participants from different region of Baghdad city, their ages ranged between (20-50) years. The person information of Each participant was asked a systematic questionnaire for the various etiological factors of genetic disease, history parents, marriage relatives, X-linked diseases such as hemophilia, G6PD dehydrogenase deficiency and color blindness. Two milliliter of blood samples was collected for DNA extraction by using commercial kit (ReliaPrep ${ }^{\mathrm{TM}}$ ) Blood gDNA Miniprep System (Promega, Madison, USA), following the instructions of the manufacturer. DNA integrity was detected using gel electrophoresis, while DNA concentration and its degree of purification was evaluated using Nanodrop.

\section{Optimization of Primer Annealing Temperature and PCR conditions}

Optimization of primer annealing temperature was performed with using gradient PCR, by using different temperatures to identify the best conditions of primer annealing using 10 Pmol primers concentration for DXS101 loci F:5'ACTCTAAATCAGTCCAAATATCT3'PET R:5'AAATCACTCCATGGCACATGTAT3'

Product size was (197-230) (6). and for loci DXS6807F:5'GAGCAATGATCTCATTTGCA3', PET, R:5'AAGTAAACATGTATAGGAAAAAGCT3' with product size (250-270) (5).

PCR amplifications with final volume of (25) $\mu 1$ of (12.5) $\mu$ l from $2 X$ GoTaq Green Master mix (Promega 2X) and $0.5 \mu \mathrm{l}$ from $10 \mathrm{pmol}$ of specific primers. Approximately $10 \mathrm{ng}$ genomic DNA was used as template. After optimize the rection conditions, PCR amplification was done with an initial incubation at $95^{\circ} \mathrm{C}$ for 11 min, followed by 27 cycles of $95^{\circ} \mathrm{C}$ for $60 \mathrm{~s}, 58.5^{\circ} \mathrm{C}$ for $60 \mathrm{~s}, 72^{\circ} \mathrm{C}$ for $3 \mathrm{~min}$, followed by a final extension for 30 $\min$ at $72^{\circ} \mathrm{C}$. Four micro-liters of the products were run on $1.5 \%$ agarose gel by electrophoresis, stained with ethidium bromide and visualized under UV light.

\section{PCR product analysis:}

Every $1 \mu \mathrm{l}$ PCR product was supplemented with twelve microliters of Hi-Di formamide (Applied Biosystems) and $0.5 \mu \mathrm{l}$ of GeneScan-500 LIZ internal size standards. Electrophoresis was carried out using the genetic analyser ABI PRIZM 310 (Applied Biosystems). The fragment sizes were calculated automatically using Genes Scan Analysis Software 3.1 (Applied Biosystems) and the results were analyzed using the Geneious Prime software. 


\section{Statistical analysis:}

The polymorphism information content (PIC) and it was suggested by (7). The power of discrimination in male (PD) and mean exclusion (MEC) was calculated according to (8) and (9). The allele's frequencies were calculated by SPSS Statistics 20.0 software. Power of Exclusion (PE), Polymorphism Information Content (PIC), Mean Exclusion Chance (MEC) and Power of Discrimination (PD) calculations were performed using http://www.chrx-str.org/.

\section{Results and discussion}

The allele frequencies and the forensic efficiency parameters of 200 sample calculated for DXS101 separately for males where by 10 alleles specified with length variation that raised in size by (3 bpincrements) ranging from (198-222) bp.As shown in Figure (1):

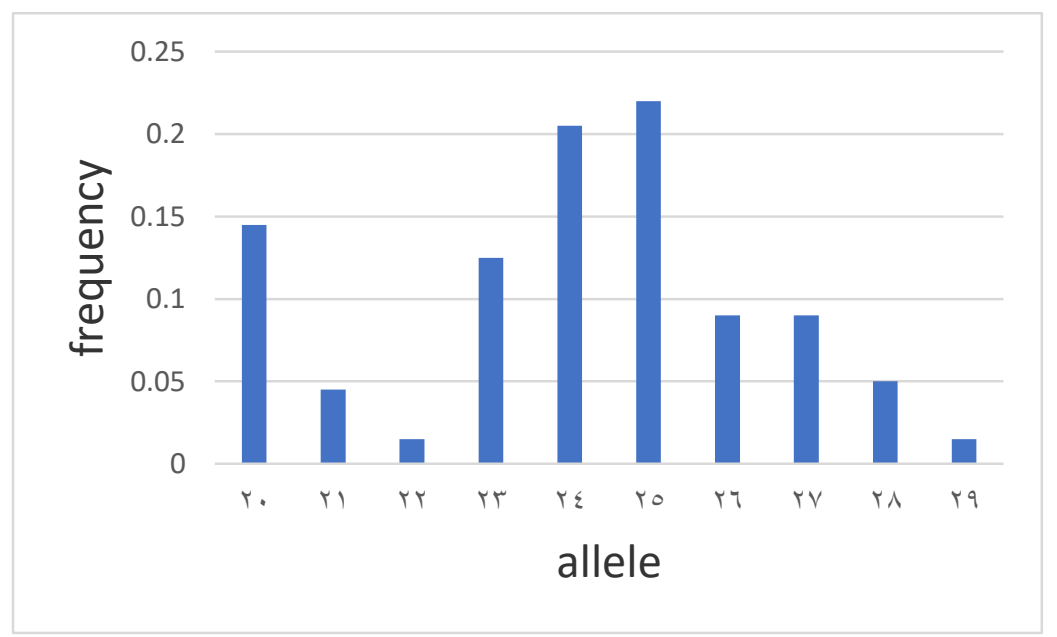

Figure (1): The percent allelefrequencyforDXS101 locus

While in previous studies perform on male Germany suggest this marker have 18 allele and rang length ranging from 179-233pb. In contras Figure $(1,2)$ and Table(1): shows the polymorphic region of DXS101loci and DXS6807 were formed by two (motifs blocks) consisting of variable numbers of trinucleotide repeat motifs block, (CTT and ATT). Alleles which are the same in size. Previous studies indicate that DXS101 is a highly informative marker that should ideally be used to measure kinship. Its length variation is caused by normal trinucleotide increment. The DXS6807 alle frequencies were measured separately for males whereby 8 alleles were detected with length variation, which increased substantially by $4 \mathrm{bp}$-increments ranging from 251-275 bp. while in previous studies perform on male Germany suggest this marker have 7 alleles and rang length ranging from 251-275pb. The polymorphic region of allele is designated according to the total number of repeat units. The repeat motifs consist from $4 \mathrm{bp}$ in motifs GATA of alleles that are identical/same in size. The previous studies suggest that DXS6807 is a suitable marker which should be used primarily for the testing of kinship. The difference in duration is caused by regular tetra nucleotide increments motifs. 


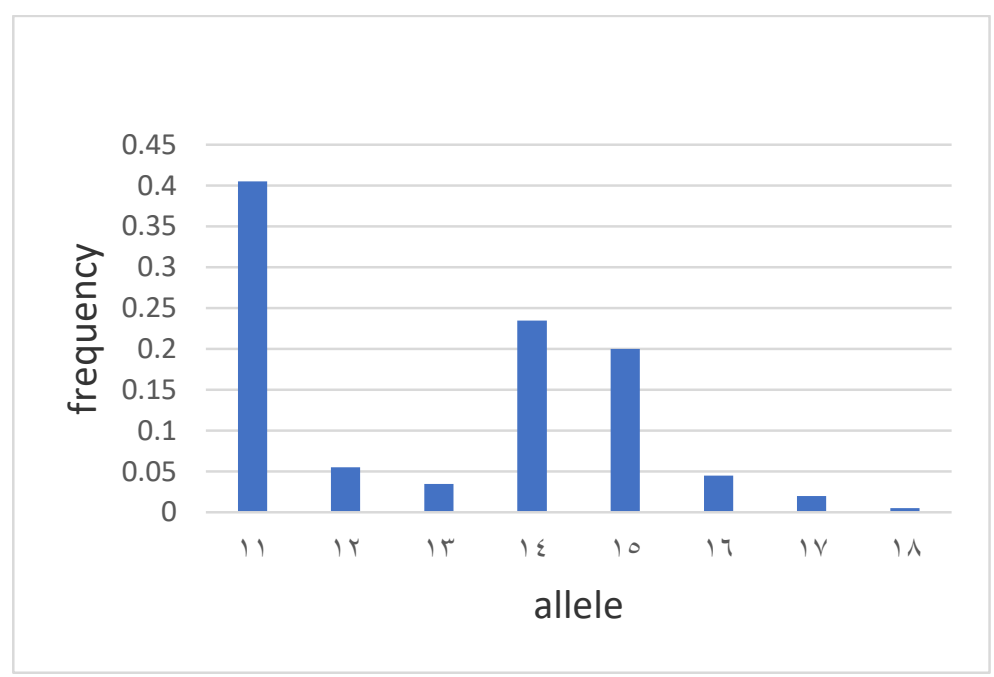

Figure (2): The percent allelefrequencyforDXS6807locus

Table (1): forensic efficiency parameters of DXS101 and DXS6807 markers

\begin{tabular}{|c|c|c|c|c|c|}
\hline Locus & $\begin{array}{c}\text { Power of } \\
\text { discrimination }\end{array}$ & $\begin{array}{c}\text { Polymorphism } \\
\text { information } \\
\text { content }\end{array}$ & $\begin{array}{c}\text { Power of } \\
\text { exclusion }\end{array}$ & $\begin{array}{c}\text { MEC } \\
\text { Kishida }\end{array}$ & $\begin{array}{c}\text { MEC } \\
\text { Krüger }\end{array}$ \\
\hline DXS101 & $\mathbf{0 . 8 5 1 7 5}$ & $\mathbf{0 . 8 3 4 7 0 8}$ & $\mathbf{0 . 6 9 8 3 1 6}$ & $\mathbf{0 . 8 3 4 7 0 8}$ & $\mathbf{0 . 7 0 3 9 4 9}$ \\
\hline DXS6807 & $\mathbf{0 . 7 3 4 0 5}$ & $\mathbf{0 . 6 9 4 8 9}$ & $\mathbf{0 . 4 8 2 8 7 9}$ & $\mathbf{0 . 6 9 4 8 9 0}$ & $\mathbf{0 . 5 1 1 6 7 9}$ \\
\hline
\end{tabular}

\section{Conclusion}

We have shown this marker DXS101 characteristic since its molecular localization and regular trinucleotides repeat structure. Besides this marker has appropriate allele distribution and stability. DXS101 is published by Edelmann and Szibor (4). DXS101was described in previous studied and exhibit not joint with HUMHPRTB and HUMARA. DXS101 located at a genetic distance ranged from around a Xp-telomere (Xp-Tel) (104.9-121) cM corresponding to (Xq21). In (33-Xq22. 3). make this marker appropriate locus for forensic application. HumDXS6807 marker characteristics since its molecular localization and regular tetra nucleotides repeat structure. Besides this markerhas appropriate allele distribution and stability. HumDXS6807 is also known GATA52B03 published by Edelmann and Szibor (5). Because HumDXS6807 is not joint with HUMHPRTB and HUMARA. HumDXS6807 located at a genetic distance ranged from around $80 \mathrm{cM}$ and $150 \mathrm{cM}$ make this marker appropriate locus for forensic application. Our result suggests that two markers more efficient in forensic application. 


\section{Reference}

1. Hameed IH, Kareem MA, Rafid HH. X-chromosome short tandem repeat, advantages and typing technology review. African Journal of Biotechnology. (2015); 14(7): 535-541.

2. Presciuttini S, Toni C, Alu M, Asmundo, et al. X-chromosome in Italy: A database of 29 STR markers. Forensic Science International: Genetics Supplement Series. (2011); 3(1): 37-38.

3. Pinto N, Gusmão L, Amorim A. X-chromosome markers in kinship testing: a generalisation of the IBD approach identifying situations where their contribution is crucial. Forensic Science International: Genetics. (2011); 5(1): 27-32.

4. Edelmann J, Szibor R. DXS101: a highly polymorphic X-linked STR. International Journal of Legal Medicine. (2001); 114(4-5): 301-304.

5. Edelmann J, Szibor R. Validation of the HumDXS6807 short tandem repeat polymorphism for forensic application. ELECTROPHORESIS: An International Journal. (1999); 20(14): 2844-2846.

6. Nakamura Y, Minaguchi K. Sixteen X-chromosomal STRs in two octaplex PCRs in Japanese population and development of 15-locus multiplex PCR system. International journal of legal medicine. (2010); 124 (5): 405-414.

7. Botstein D, White RL, Skolnick M, et al. Construction of a genetic linkage map in man using restriction fragment length polymorphisms. American journal of human genetics. (1980); 32(3): 314.

8. KRÜGER J, et al. Zur Verwendung des Polymorphismus der sauren Erythrocytenphosphatase bei der Vaterschaftsbegutachtung. Deutsche Zeitschrift für die gesamte gerichtliche Medizin. (1968); 64(2): 127146.

9. KISHIDA T, et al. Duplex PCR of the Y-27H39 and HPRT loci with reference to Japanese population data on the HPRT locus. Nihon hoigaku zasshi= The Japanese journal of legal medicine. (1997); 51(2): 6769. 